\title{
Chattering-Free Sliding-Mode Control for Electromechanical Actuator with Backlash Nonlinearity
}

\author{
Dongqi Ma, Hui Lin, and Bingqiang Li \\ School of Automation, Northwestern Polytechnical University, Xi'an 710129, China \\ Correspondence should be addressed to Dongqi Ma; madongqi0499@163.com
}

Received 9 October 2016; Accepted 22 January 2017; Published 13 February 2017

Academic Editor: Ephraim Suhir

Copyright (C) 2017 Dongqi Ma et al. This is an open access article distributed under the Creative Commons Attribution License, which permits unrestricted use, distribution, and reproduction in any medium, provided the original work is properly cited.

\begin{abstract}
Considering the backlash nonlinearity and parameter time-varying characteristics in electromechanical actuators, a chatteringfree sliding-mode control strategy is proposed in this paper to regulate the rudder angle and suppress unknown external disturbances. Different from most existing backlash compensation methods, a special continuous function is addressed to approximate the backlash nonlinear dead-zone model. Regarding the approximation error, unmodeled dynamics, and unknown external disturbances as a disturbance-like term, a strict feedback nonlinear model is established. Based on this nonlinear model, a chattering-free nonsingular terminal sliding-mode controller is proposed to achieve the rudder angle tracking with a chattering elimination and tracking dynamic performance improvement. A Lyapunov-based proof ensures the asymptotic stability and finitetime convergence of the closed-loop system. Experimental results have verified the effectiveness of the proposed method.
\end{abstract}

\section{Introduction}

Electromechanical actuator is an important part of classical servo control systems [1], and it is widely used in the aerospace, military, transportation, and some other fields, such as aircraft servo systems [2], missile seeker servo platforms [3], and aircraft and vehicle braking systems [4]. As important techniques of electromechanical actuators, high performance servo motor and advanced stable controller are research hotspots in recent years. Permanent Magnet Synchronous Motor (PMSM) has many advantages like small torque and speed ripple, high torque-inertia ratio, and wide speed range. PMSM now is widely used in industries, especially in the aerospace [5].

Backlash is an important nonlinearity that limits the dynamic performance and steady precision of speed and position control in industrial, automation, and other applications. It exists in every mechanical system where a driving subsystem is not directly connected with the driven subsystem. Different from traditional hydraulic and pneumatic actuators, EMA has gear reducer which leads to the backlash nonlinearity. Backlash nonlinearity causes delays, noise, and oscillations which affect the system dynamic performance and steady precision. Due to the dynamic, nondifferentiable backlash nonlinearity and because it is difficult to be accurately measured, the compensation control is difficult to be designed.

Recently, studies of nonlinear systems with backlash have been the research hotspot. The noncontinuous transfer relationship caused by the backlash can be described from different perspectives. A number of mathematical models have been presented, such as hysteresis model $[6,7]$, deadzone model [8], and impact-damper model [9]. The hysteresis model describes the relationship between the output angle of backlash and the input angle under the assumption that the shaft is stiff [10]. Dead-zone model describes the torque transitive relationship between the driving and driven subsystem [11]. Impact-damper model reflects the mechanism in the process of impaction caused by backlash.

Building a backlash inverse model at the control input and designing the feed forward compensation to offset the impact of backlash are most widely used in the current control compensation strategy. In practical engineering, PID control is the most commonly used algorithm due to its simple structure, but it is difficult to deal with nonlinear systems. So many intelligent methods have been studied 
including robust control [12], adaptive backstepping control [6], model predictive control [13], fuzzy control [14], and sliding-mode control [15]. This strategy is often applied in the system in which the backlash locates at the input or output. However, for the EMA, backlash cannot be simply converted to the control input. Inverse model compensation is no longer applicable. It is more reasonable to use the backlash dead-zone model for describing the force transfer relationship. Then the nonlinear system containing backlash can be regarded as Nonsmooth Sandwich System [16, 17].

Reference [8] uses the optimal control approach and adopts different control strategies at the backlash working time and normal condition, which can both make the backlash compensation and reduce the impact of backlash on the system. But the controller structure is too complex to be implemented. Reference [18] takes the differentiable function to approximate the dead-zone model and design a backstepping controller. The design requires an accurate system model while the system parameters are time-variant and are difficult to be accurately obtained, such as the stiffness coefficient and the width of the backlash. Reference [19] uses a fuzzy function approaching nonlinear function created by the backstepping strategy to simplify the design. The disadvantage is that they use an approximating function and its derivative term as the state variables. The states of the system do not have a clear physical significance and cannot be directly measured. And the control quality depends on the control parameter settings. So the backlash compensation effect cannot be guaranteed. Reference [20] designs an adaptive sliding-mode control (ASMC) based on extended state observer (ESO). ESO is employed to estimate the system states and an adaptive law is adopted to compensate backlash. The selected sliding surface and the controller can only guarantee the convergence but not terminal. And the chattering phenomenon has not been well solved.

Sliding-mode control (SMC) has self-adaptability to the system uncertainties and disturbance. But the singularity, chattering, and the convergence speed limit the application of SMC. In this paper, a chattering-free nonsingular fast terminal sliding-mode control (CNFTSMC) is presented based on backstepping method in order to compensate the backlash nonlinearity of the EMA and reduce the influence of the unknown external disturbance and parameter variation on the system. Compared to the existing methods, it solved the singularity problem by the design of the sliding surface and the control input. And a smooth control method via the low-pass filter is developed. This method can reduce the impact caused by the backlash effectively.

\section{The Mathematical Model of EMA with Backlash}

Several hypotheses are considered as follows before establishing the model:
(3) The three phases are symmetric.

(4) The air gap magnetic field is a sine wave.

The mathematical model of PMSM in $d q$ axis is displayed as follows:

$$
\begin{aligned}
& \frac{d \theta}{d t}=\omega \\
& \frac{d \omega}{d t}=\frac{3 p \varphi_{f}}{2 J} i_{q}-\frac{B}{J} \omega-\frac{T_{L}}{J} \\
& \frac{d i_{q}}{d t}=-\frac{R}{L} i_{q}-p \omega i_{d}-\frac{p \varphi_{f}}{L} \omega+\frac{u_{q}}{L} \\
& \frac{d i_{d}}{d t}=-\frac{R}{L} i_{d}+p \omega i_{q}+\frac{u_{d}}{L},
\end{aligned}
$$

where $\theta$ and $\omega$ are the rotor angle and speed, respectively; $\varphi_{f}$ is the permanent magnet flux; $p$ is the pole pairs; $J$ is the moment of inertia of the PMSM; $B$ is the viscous friction coefficient; $u_{d}$ and $u_{q}$ are the voltages in $d q$ axis; $i_{d}$ and $i_{q}$ are the currents in $d q$ axis; $R$ is the phase winding resistance; $L$ is the inductance; $T_{L}$ is the load torque.

The dynamic equation of the driven subsystem is shown by

$$
\begin{aligned}
\frac{d \theta_{s}}{d t} & =\omega_{s} \\
J_{s} \frac{d \omega_{s}}{d t} & =\eta T_{L}-B_{s} \omega_{s}-T_{s},
\end{aligned}
$$

where $\theta_{s}$ and $\omega_{s}$ are the angle and speed of the load mechanism, respectively; $J_{s}$ and $B_{s}$ are the moment of inertia and the friction coefficient of the load mechanism; $\eta$ is the reduction ratio; $T_{s}$ is the disturbance torque which includes the coupling torque and the unknown external disturbance.

There is a time-delay in torque transfer from the driving subsystem to the driven subsystem because of the backlash which exists in the reducer. The dead-zone model of the backlash is described by

$$
T_{L}= \begin{cases}k_{T}(\Delta \theta-\alpha) & \text { if } \Delta \theta>\alpha \\ 0 & \text { if }|\Delta \theta| \leq \alpha \\ k_{T}(\Delta \theta+\alpha) & \text { if } \Delta \theta<-\alpha,\end{cases}
$$

where $\Delta \theta=\theta-\eta \theta_{s}$ is the relative angular displacement; $2 \alpha$ is the width of the backlash; $k_{T}$ is the gear stiffness coefficient. In order to overcome the nonsmooth property of the model in (3), a differentiable function is adopted to approximate $T_{L}$, given by

$$
T_{f}=k_{T} \Delta \theta+\frac{k_{T}}{2 h} \ln \left(\frac{e^{h(\Delta \theta-\alpha)}+e^{-h(\Delta \theta-\alpha)}}{e^{h(\Delta \theta+\alpha)}+e^{-h(\Delta \theta+\alpha)}}\right),
$$

(2) Ignore the hysteresis losses and vortex losses. 

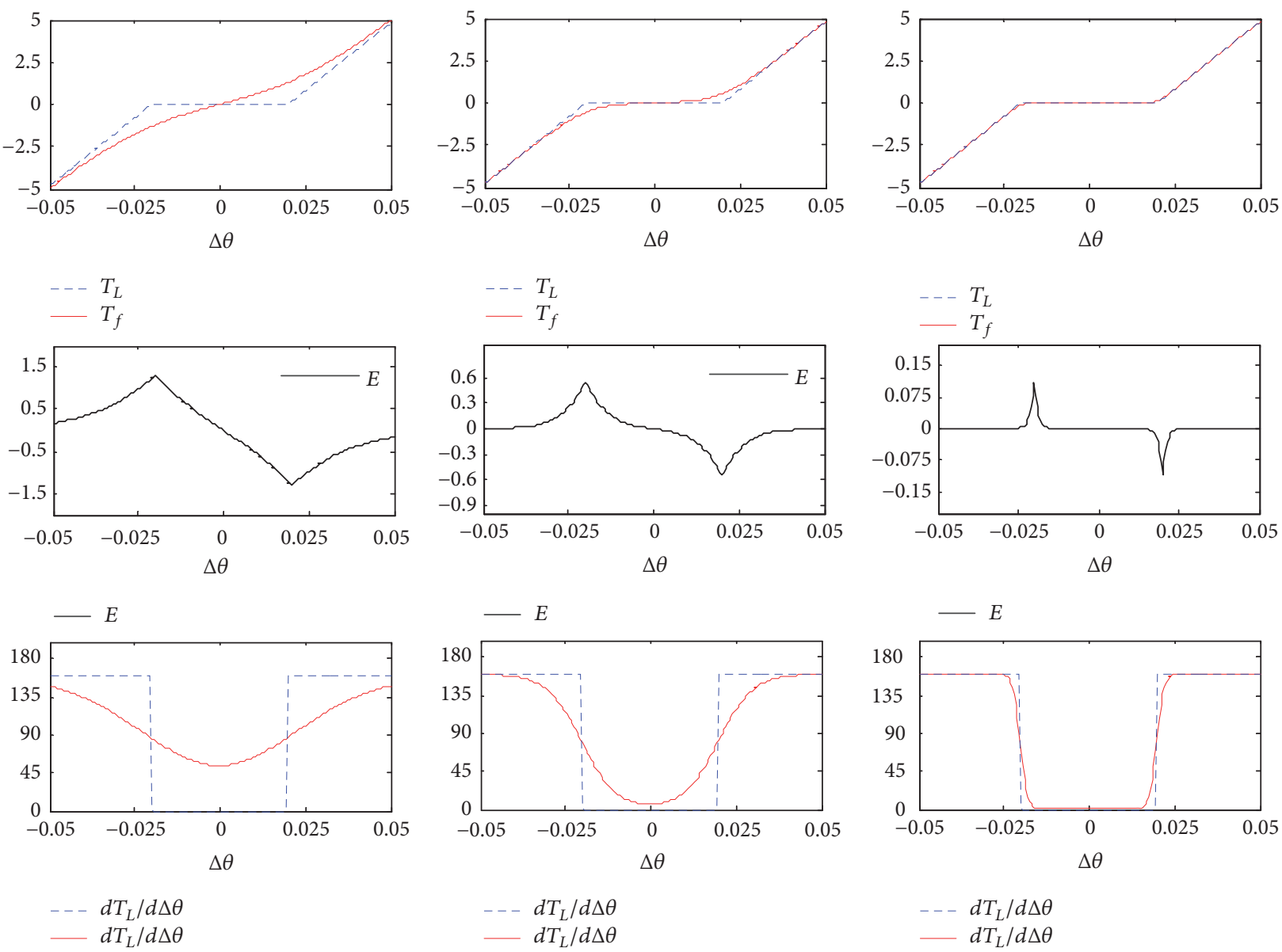

(a)

$---d T_{L} / d \Delta \theta$
$-d T_{L} / d \Delta \theta$

(b)

(c)

FIgURE 1: Approximation of dead-zone with different smooth degrees: $k_{T}=160, \alpha=0.02$. (a) $h=40$, (b) $h=100$, and (c) $h=500$.

where $h$ is a positive parameter (refer to as smooth degree). And the approximation error can be derived as

$$
\begin{aligned}
E(\Delta \theta)= & T_{L}-T_{f} \\
& = \begin{cases}-k_{T} \alpha-\frac{k_{T} \tau(\Delta \theta)}{2 h} & \text { if } \Delta \theta>\alpha \\
-k_{T} \Delta \theta-\frac{k_{T} \tau(\Delta \theta)}{2 h} & \text { if }|\Delta \theta| \leq \alpha \\
k_{T} \alpha-\frac{k_{T} \tau(\Delta \theta)}{2 h} & \text { if } \Delta \theta<-\alpha,\end{cases}
\end{aligned}
$$

where $\tau(\Delta \theta)=\ln \left(\left(e^{h(\Delta \theta-\alpha)}+e^{-h(\Delta \theta-\alpha)}\right) /\left(e^{h(\Delta \theta+\alpha)}+e^{-h(\Delta \theta+\alpha)}\right)\right)$.

Remark 1. We can obtain from [18] that $|E(\Delta \theta)|<k_{T} \ln 2 / 2 h$ and $\lim _{h \rightarrow+\infty} E(\Delta \theta)=0$, which means the nonsmooth property of backlash can be smoothed to any arbitrary precision by model (4). Thus, $h$ in (4) is referred to as "smooth degree," as illustrated in Figure 1.
Then we can get from (1), (2), (3), (4), and (5):

$$
\begin{aligned}
& \frac{d \omega_{s}}{d t} \\
& \quad=J_{s}^{-1}\left(-\eta^{2} k_{T} \theta_{s}+\eta k_{T} \theta-B_{s} \omega_{s}+\frac{k_{T}}{2 h} \tau(\Delta \theta)\right)+d_{1} \\
& \frac{d \omega}{d t} \\
& =J^{-1}\left(\eta k_{T} \theta_{s}-k_{T} \theta-B \omega+1.5 p \varphi_{f} i_{q}-\frac{k_{T}}{2 h} \tau(\Delta \theta)\right) \\
& \quad+d_{2},
\end{aligned}
$$

where $d_{1}=J_{s}^{-1}\left(\eta E(\Delta \theta)-T_{s}\right)$ and $d_{2}=-J^{-1} E(\Delta \theta)$ which are called the disturbance-like terms.

Define the system state variables as $\mathbf{x}=\left[\theta_{s}, \omega_{s}, \theta, \omega, i_{q}, i_{d}\right]$; then the mathematical model of EMA with backlash is shown as

$$
\frac{d \theta_{s}}{d t}=\omega_{s}
$$




$$
\begin{aligned}
\frac{d \omega_{s}}{d t}= & J_{s}^{-1}\left(-\eta^{2} k_{T} \theta_{s}-B_{s} \omega_{s}+\frac{k_{T}}{2 h} \tau(\Delta \theta)\right)+J_{s}^{-1} \eta k_{T} \theta \\
& +d_{1} \\
\frac{d \theta}{d t}= & \omega \\
\frac{d \omega}{d t}= & J^{-1}\left(\eta k_{T} \theta_{s}-k_{T} \theta-B \omega-\frac{k_{T}}{2 h} \tau(\Delta \theta)\right) \\
& +1.5 J^{-1} p \varphi_{f} i_{q}+d_{2} \\
y= & \theta_{s},
\end{aligned}
$$

where $y$ is the system output.

Two assumptions are listed as follows for the subsequent analysis.

Assumption 2. The given signals $\theta_{s}(t), \ddot{\theta}_{s}^{*}(t)$, and $\dddot{\theta}_{s}^{*}(t)$ are bounded and continuous.

Assumption 3. The disturbances $d_{i}(i=1,2)$ and their derivatives are bounded which means that there are constants $l_{d i}$ and $\rho_{i}$ satisfying the inequations: $\left|d_{i}\right| \leq l_{d i},\left|\dot{d}_{i}\right| \leq \rho_{i}$.

\section{Design of the Control}

3.1. Design the Control for the Driven Subsystem. Define the error variables $e_{1}=\theta_{s}-\theta_{s}^{*}$ and $e_{2}=\dot{e}_{1}=\omega_{s}-\dot{\theta}_{s}^{*}$. The slidingmode surface can be written as

$$
\begin{aligned}
& \sigma_{1}=e_{1}+\lambda_{1} e_{2} \\
& s_{1}=\sigma_{1}+\beta_{11} \operatorname{sgn}\left(\sigma_{1}\right)\left|\sigma_{1}\right|^{\gamma_{11}}+\beta_{12} \operatorname{sgn}\left(\dot{\sigma}_{1}\right)\left|\dot{\sigma}_{1}\right|^{\gamma_{12}} .
\end{aligned}
$$

And the virtual control $\theta^{*}$ is shown:

$$
\begin{aligned}
\theta^{*} & =J_{s} \eta^{-1} k_{T}^{-1}\left(\theta_{e q}^{*}+\theta_{s w}^{*}\right) \\
\theta_{e q}^{*} & =-J_{s}^{-1}\left(-\eta^{2} k_{T} \theta_{s}-B_{s} \omega_{s}+4 \eta k_{T} \alpha \tau(\Delta \theta)\right)+\ddot{\theta}_{s}^{*} \\
& +\lambda_{1} \ddot{\theta}_{s}^{*} \\
\theta_{s w}^{*} & +\lambda_{1} \dot{\theta}_{s w}^{*}=v_{1} \\
v_{1} & =-\left[\left(\beta_{12} \gamma_{12}\right)^{-1} \operatorname{sgn}\left(\dot{\sigma}_{1}\right)\left|\dot{\sigma}_{1}\right|^{2-\gamma_{12}}\right. \\
& \cdot\left(1+\beta_{11} \gamma_{11}\left|\sigma_{1}\right|^{\gamma_{11}-1}\right)+k_{1} s_{1}+\left(k_{d 1}+k_{l 1}+k_{n 1}\right) \\
& \left.\cdot \operatorname{sgn}\left(s_{1}\right)\right] .
\end{aligned}
$$

Select the Lyapunov function as follows:

$$
V_{1}=\frac{1}{2} s_{1}^{2}
$$

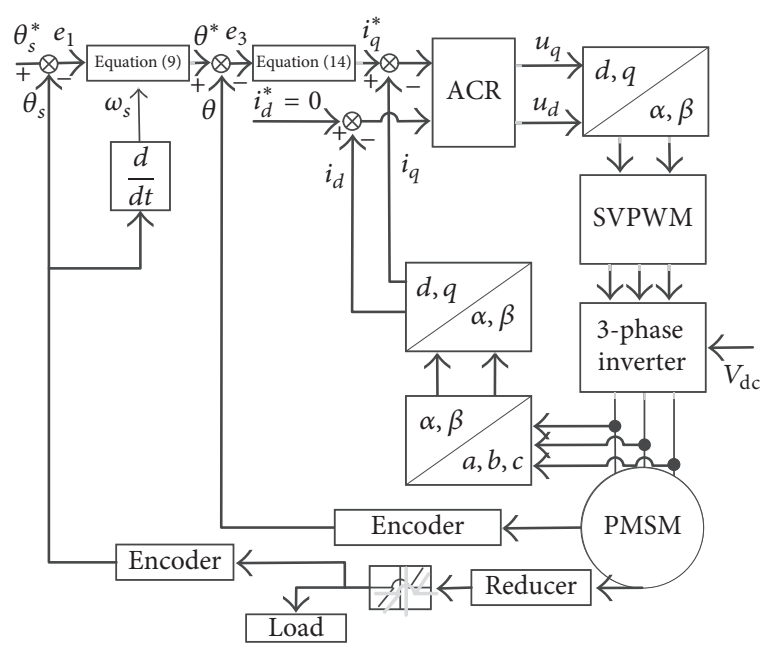

Figure 2: Control block diagram of the system.

We can obtain

$$
\begin{aligned}
\dot{V}_{1} & =s_{1} \dot{s}_{1}=\beta_{12} \gamma_{12}\left|\dot{\sigma}_{1}\right|^{\gamma_{12}-1} s_{1}\left(-k_{1} s_{1}\right. \\
& \left.-\left(k_{d 1}+k_{l 1}+k_{n 1}\right) \operatorname{sgn}\left(s_{1}\right)+d_{1}+\lambda_{1} \dot{d}_{1}\right) \\
& \leq \beta_{12} \gamma_{12}\left|\dot{\sigma}_{1}\right|^{\gamma_{12}-1} s_{1}\left(-k_{1} s_{1}-k_{n 1} \operatorname{sgn}\left(s_{1}\right)\right) \\
& =-K_{11} s_{1}^{2}-K_{12}\left|s_{1}\right|=-2 K_{11} V_{1}-\sqrt{2} K_{12} V_{1}^{1 / 2} \leq 0 .
\end{aligned}
$$

And $\dot{V}_{1}<0$ for $\left|s_{1}\right| \neq 0$, where $K_{11}=k_{1} \beta_{12} \gamma_{12}\left|\dot{\sigma}_{1}\right|^{\gamma_{12}-1}$ and $K_{12}=k_{n 1} \beta_{12} \gamma_{12}\left|\dot{\sigma}_{1}\right|^{\gamma_{12}-1}$.

3.2. Design the Control for the Driving Subsystem. Define the error variables $e_{3}=\theta-\theta^{*}$ and $e_{4}=\dot{e}_{3}=\omega-\dot{\theta}^{*}$; the slidingmode surface and the controller are obtained as

$$
\begin{aligned}
\sigma_{2} & =e_{3}+\lambda_{2} e_{4} \\
s_{2} & =\sigma_{2}+\beta_{21} \operatorname{sgn}\left(\sigma_{2}\right)\left|\sigma_{2}\right|^{\gamma_{21}}+\beta_{22} \operatorname{sgn}\left(\dot{\sigma}_{2}\right)\left|\dot{\sigma}_{2}\right|^{\gamma_{22}} \\
i_{q}^{*} & =\frac{2}{3} J p^{-1} \varphi_{f}^{-1}\left(i_{q e q}^{*}+i_{q s w}^{*}\right) \\
i_{q e q}^{*} & =-J^{-1}\left(\eta k_{T} \theta_{s}-k_{T} \theta-B \omega-4 \eta k_{T} \alpha \tau(\Delta \theta)\right)+\ddot{\theta}^{*} \\
& +\lambda_{2}{ }^{*} \\
i_{q s w}^{*} & +\lambda_{2} i_{q s w}^{*}=v_{2} \\
v_{2}= & -\left[\left(\beta_{22} \gamma_{22}\right)^{-1} \operatorname{sgn}\left(\dot{\sigma}_{2}\right)\left|\dot{\sigma}_{2}\right|^{2-\gamma_{22}}\right. \\
& \cdot\left(1+\beta_{21} \gamma_{21}\left|\sigma_{2}\right|^{\gamma_{21}-1}\right)+k_{2} s_{2}+\left(k_{d 2}+k_{l 2}+k_{n 2}\right) \\
& \left.\cdot \operatorname{sgn}\left(s_{2}\right)\right] .
\end{aligned}
$$

In order to realize the decoupling of current and speed and simplify the controller, the control strategy $i_{d}^{*}=0$ has been used. Now the control block diagram of the system is displayed in Figure 2. 


\subsection{Stability Analysis}

Lemma 4. The equilibrium point $x=0$ is globally finite-time stable for any given initial condition $x(0)=x_{0}$ if a Lyapunov description can be obtained as [21]

$$
\dot{V}+m_{1} V+m_{2} V^{\kappa} \leq 0 .
$$

And then the setting time can be given by

$$
t \leq t_{0}+\frac{1}{m_{1}(1-\kappa)} \ln \frac{m_{1} V^{1-\kappa}\left(t_{0}\right)+m_{2}}{m_{2}},
$$

where $m_{1}, m_{2}>0,0<\kappa<1$.

Lemma 5. Assume $a_{1}, a_{2}, \ldots, a_{n}$ and $\delta \in(0,2)$ are all positive numbers; then the following inequality holds [22]:

$$
\left|a_{1}\right|^{\delta}+\left|a_{2}\right|^{\delta}+\cdots+\left|a_{n}\right|^{\delta} \geq\left(a_{1}^{2}+a_{2}^{2}+\cdots+a_{n}^{2}\right)^{\delta / 2} .
$$

Theorem 6. For system (7), if the controllers are designed as (9) and (14), then the system will converge in finite-time. And the settling time is given by (21).

Proof. Consider the following Lyapunov function:

$$
V_{M}=V_{1}+\frac{1}{2} s_{2}^{2}
$$

Then the derivative of $V_{M}$ is

$$
\begin{aligned}
\dot{V}_{M} & =\dot{V}_{1}+\dot{V}_{2}=s_{1} \dot{s}_{1}+s_{2} \dot{s}_{2}=\beta_{12} \gamma_{12}\left|\dot{\sigma}_{1}\right|^{\gamma_{12}-1} \\
& \cdot s_{1}\left(-k_{1} s_{1}-\left(k_{d 1}+k_{l 1}+k_{n 1}\right) \operatorname{sgn}\left(s_{1}\right)+d_{1}\right. \\
& \left.+\lambda_{1} \dot{d}_{1}\right)+\beta_{22} \gamma_{22}\left|\dot{\sigma}_{2}\right|^{\gamma_{22}-1} s_{2}\left(-k_{2} s_{2}\right. \\
& \left.-\left(k_{d 2}+k_{l 2}+k_{n 2}\right) \operatorname{sgn}\left(s_{2}\right)+d_{2}+\lambda_{2} \dot{d}_{2}\right) \\
& \leq \beta_{12} \gamma_{12}\left|\dot{\sigma}_{1}\right|^{\gamma_{12}-1} s_{1}\left(-k_{1} s_{1}-k_{n 1} \operatorname{sgn}\left(s_{1}\right)\right) \\
& +\beta_{22} \gamma_{22}\left|\dot{\sigma}_{2}\right|^{\gamma_{22}-1} s_{2}\left(-k_{2} s_{2}-k_{n 2} \operatorname{sgn}\left(s_{2}\right)\right) \\
& =-K_{11} s_{1}^{2}-K_{12}\left|s_{1}\right|-K_{21} s_{2}^{2}-K_{22}\left|s_{2}\right| \leq-\sum_{i=1}^{2} K_{i 1} s_{i}^{2} \\
& -\sum_{i=1}^{2} K_{i 2}\left|s_{i}\right|
\end{aligned}
$$

where $K_{21}=k_{2} \beta_{22} \gamma_{22}\left|\dot{\sigma}_{2}\right|^{\gamma_{22}-1}, K_{22}=k_{n 2} \beta_{22} \gamma_{22}\left|\dot{\sigma}_{2}\right|^{\gamma_{22}-1}, \bar{K}_{1}=$ $\min \left\{K_{i 1}\right\}$, and $\bar{K}_{2}=\min \left\{K_{i 2}\right\}$.

From Lemma 5, (19) can be rewritten as

$$
\dot{V}_{M} \leq-\bar{K}_{1} \sum_{i=1}^{2} s_{i}^{2}-\bar{K}_{2} \sum_{i=1}^{2}\left|s_{i}\right| \leq-2 \bar{K}_{1} V_{M}-\sqrt{2} \bar{K}_{2} V_{M}^{1 / 2}
$$

According to Theorem 6 and Lemma 4, the system will converge in finite-time:

$$
t_{M} \leq t_{M 0}+\frac{1}{\bar{K}_{1}} \ln \frac{2 \bar{K}_{1} V_{M}\left(t_{M 0}\right)^{1 / 2}+\sqrt{2} \bar{K}_{2}}{\sqrt{2} \bar{K}_{2}} .
$$

TABLE 1: Parameters of the system.

\begin{tabular}{lccc}
\hline Para & Description & Value & Unit \\
\hline$p$ & Number of pole pairs & 5 & - \\
$\varphi_{f}$ & Permanent magnet flux linkage & 0.143 & $\mathrm{~Wb}$ \\
$J$ & Moment of inertia of PMSM & $2.8 \times 10^{-4}$ & $\mathrm{~kg} \cdot \mathrm{m}^{2}$ \\
$B$ & Viscous friction coefficient & $1.0 \times 10^{-4}$ & $\mathrm{~N} \cdot \mathrm{m} / \mathrm{rad} / \mathrm{s}$ \\
$R$ & Stator resistance & 1.73 & $\Omega$ \\
$L$ & Inductance & 7 & $\mathrm{mH}$ \\
$T_{L}$ & Rated torque & 4.7 & $\mathrm{~N} \cdot \mathrm{m}$ \\
$J_{s}$ & Moment of inertia of flap & 0.4 & $\mathrm{~kg} \cdot \mathrm{m}^{2}$ \\
$B_{s}$ & Friction coefficient of flaps & 0.12 & $\mathrm{~N} \cdot \mathrm{m} / \mathrm{rad} / \mathrm{s}$ \\
$\eta$ & Gear reduction ratio & 30 & - \\
$V_{\mathrm{dc}}$ & DC link voltage & 270 & $\mathrm{~V}$ \\
$k_{T}$ & Stiffness coefficient & 160 & $\mathrm{~N} \cdot \mathrm{m} / \mathrm{rad} / \mathrm{s}$ \\
$2 \alpha$ & Width of the backlash & 0.04 & $\mathrm{rad}$ \\
\hline
\end{tabular}

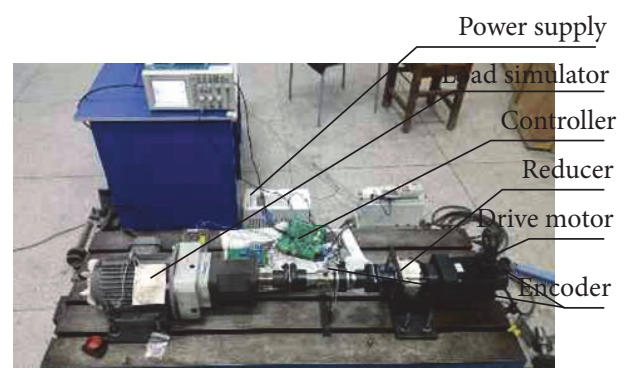

FIGURE 3: The experimental platform.

Remark 7. In Theorem 6, because of the switching function $\operatorname{sgn}(\cdot), v$ is nonsmooth. Let $v$ pass a low-pass filter, and $u_{s w}$ is the output of the filter. So the switching control term $u_{s w}$ is smooth. If the switching control term $u_{s w}$ is designed as $\dot{u}_{s w}=v$, it is also a smooth function. But it is more difficult to be implemented in engineering applications.

\section{Experimental Studies}

In order to demonstrate the effectiveness of the proposed method, the platform shown in Figure 3 has been built which is controlled by TMS320F28335 of TI.

The platform is mainly composed of power supply, controller, drive motor, reducer, and load simulator. The parameters of the system are displayed in Table 1.

The parameters of the sliding-mode surface $\lambda_{1}=\lambda_{2}=$ $0.1, \beta_{11}=\beta_{21}=10, \beta_{12}=\beta_{22}=0.4, \gamma_{11}=\gamma_{21}=2, \gamma_{12}=\gamma_{22}=$ 1.5 , and the parameters of the control $k_{1}=120, k_{2}=400$, $k_{d 1}+k_{l 1}+k_{n 1}=20, k_{d 2}+k_{l 2}+k_{n 2}=35$, and the smooth degree $h=200$. The experiments are under PID control and the proposed method, respectively.

4.1. Set the Reference Signal $\theta_{s}^{*}(t)=1(\mathrm{rad})$. First, the step response of the closed-loop system has been considered and the performance is shown as Figures 4-7. 


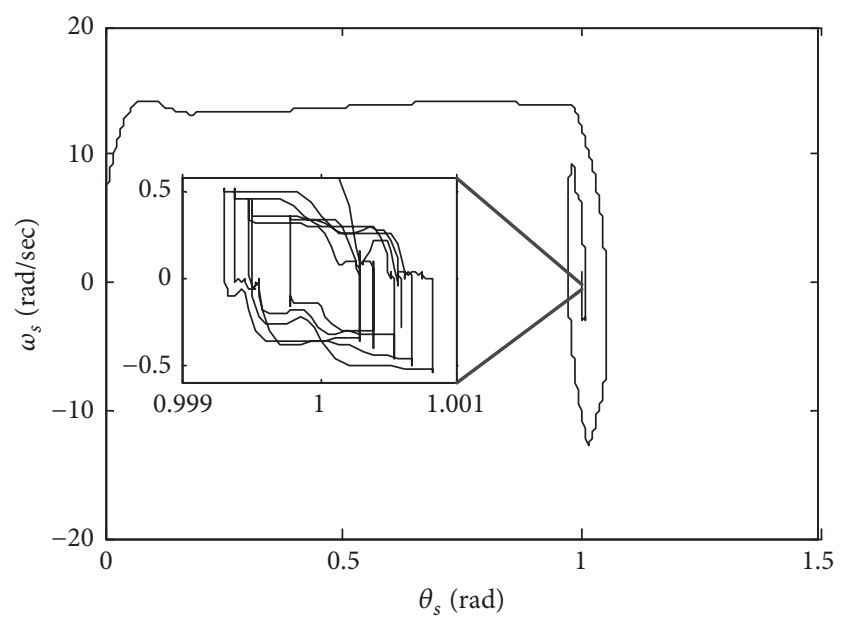

FIgURE 4: The phase trajectory under PID-controller.

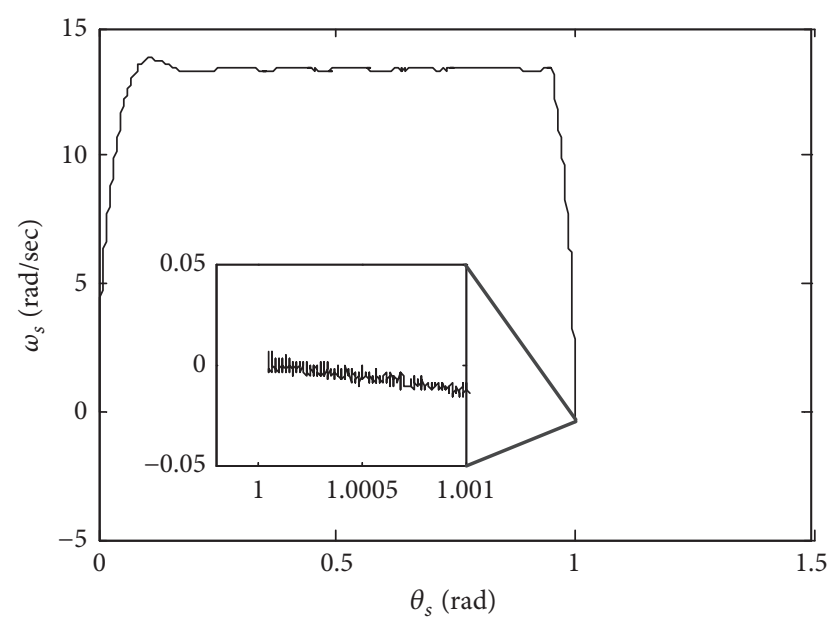

Figure 5: The phase trajectory under SMC-controller.

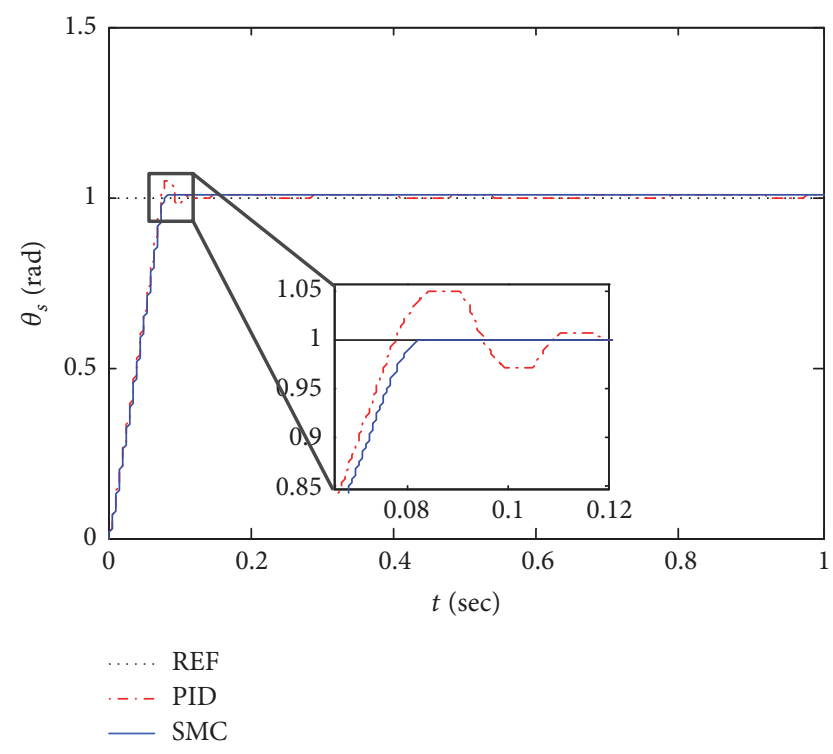

Figure 6: The tracking trajectory.

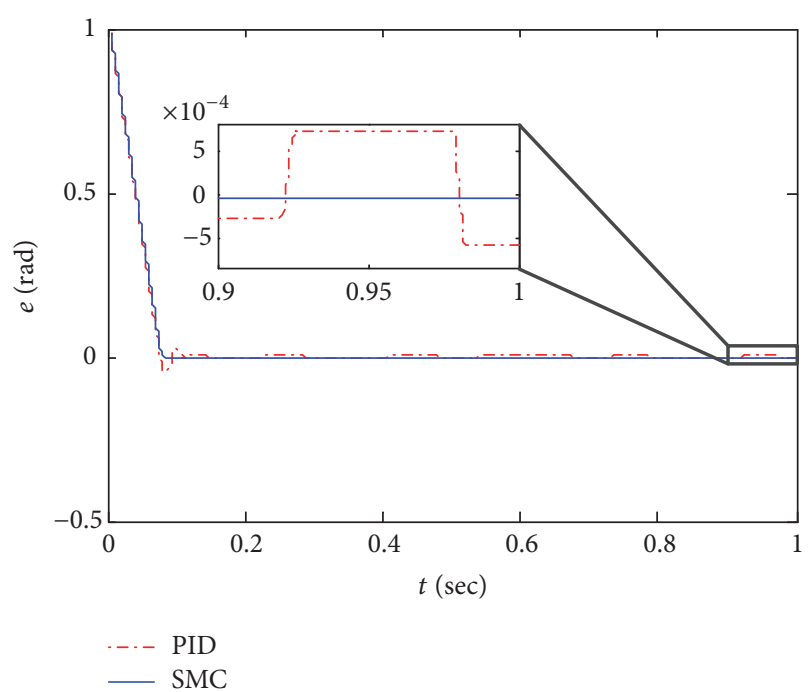

FIGURE 7: The tracking error.

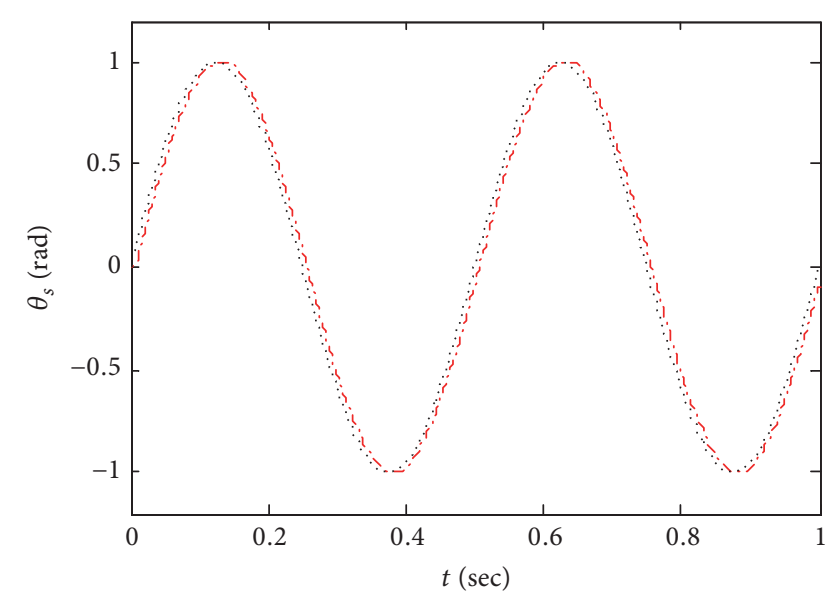

.... REF

-.. PID

FIGURE 8: The tracking trajectory under PID-controller.

4.2. Set the Reference Signal $\theta_{s}^{*}(t)=\sin (4 \pi t)(\mathrm{rad})$. The sinusoidal response is presented in Figures 8-11.

Figures 4 and 5 show the phase plane of the system. We can obtain that the proposed control can eliminate the limit cycle phenomenon. Figure 6 shows that the response under CNFTSMC has a smaller overshoot than that under PID-controller. And we can see from Figure 7 that the proposed controller has a higher tracking accuracy. Figures 8-11 illustrate that the proposed controller can reduce the phase delay and the tracking error.

\section{Conclusion}

A chattering-free nonsingular terminal sliding-mode control based on backstepping has been proposed to achieve the rudder angle tracking for EMA with considering the backlash 


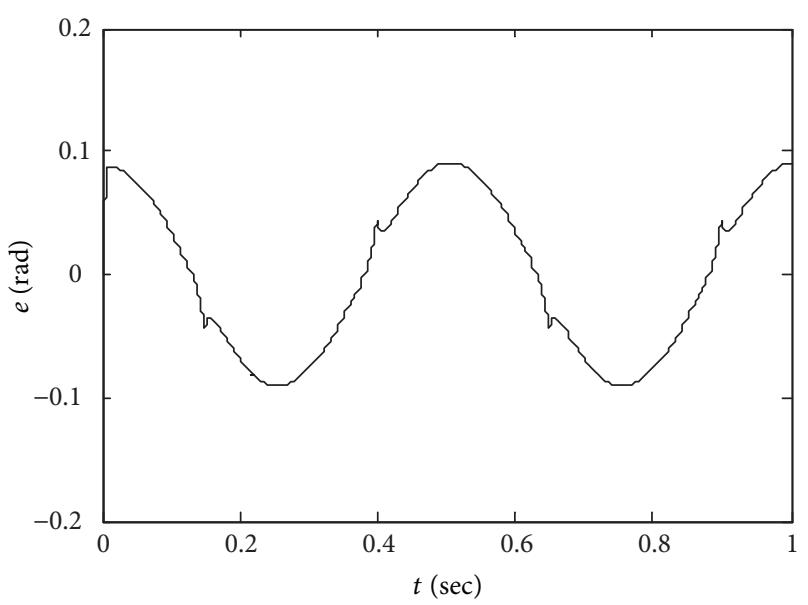

FIgURE 9: The tracking error under PID-controller.

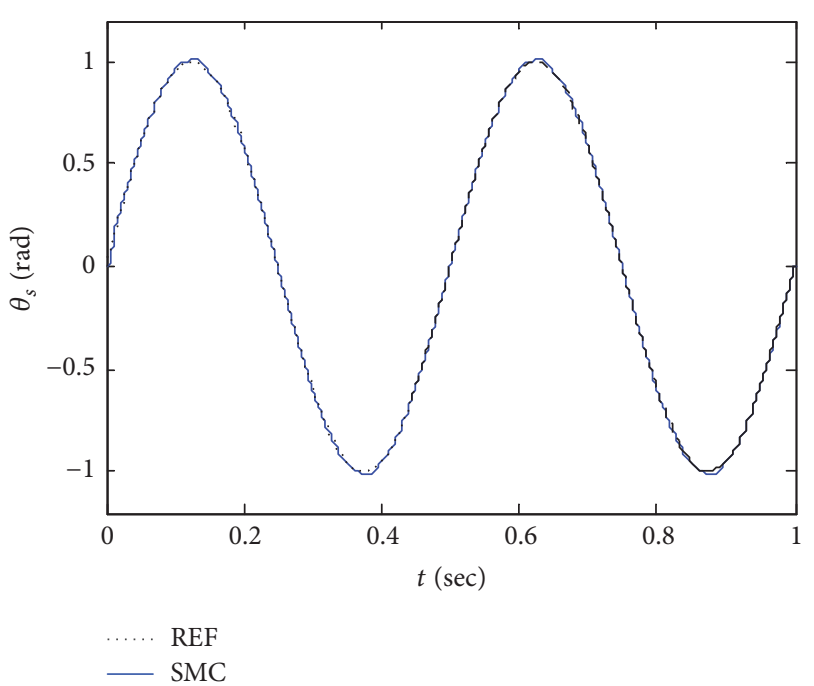

Figure 10: The tracking trajectory under SMC-controller.

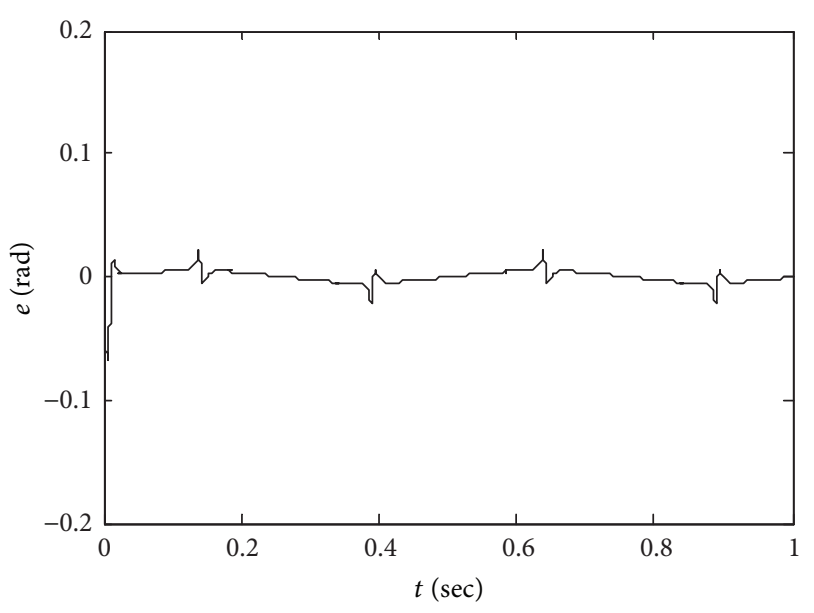

FIGURE 11: The tracking error under SMC-controller. nonlinearity. Our contributions are as follows:

(1) Some deficiencies of the backlash compensation control in the current studies have been overcome. The proposed method can reduce the impact caused by the backlash. The experimental results demonstrate the effectiveness of the proposed method.

(2) The singularity and chattering of conventional terminal sliding-mode control are effectively solved. The parameters can be set flexibly according to the actual demand.

(3) The system can converge in finite-time under the chattering-free nonsingular fast terminal slidingmode control.

\section{Competing Interests}

The authors declare that there is no conflict of interests regarding the publication of this article.

\section{Acknowledgments}

This research was supported by the National Natural Science Fund of China (Grant 51407143).

\section{References}

[1] H. Guo and W. Xing, "Development of electromechanical actuators," Chinese Journal of Aeronautics, vol. 28, no. 3, pp. 620627, 2007.

[2] H. Qi, Y. Fu, X. Qi, and Y. Lang, "Architecture optimization of more electric aircraft actuation system," Chinese Journal of Aeronautics, vol. 24, no. 4, pp. 506-513, 2011.

[3] S.-Y. Lee and J.-S. Lee, "A control for 2-axis gimbaled millimeter wave seeker using space vector PWM of PMSM," Transactions of the Korean Institute of Electrical Engineers, vol. 60, no. 12, pp. 2386-2391, 2011.

[4] Q. H. Zhang and Y. R. Li, "Design and analysis on aircraft brake system," Aeronautical Computer Technique, vol. 33, no. 3, pp. 97100, 2003.

[5] Z. Wen, Z. Wang, X. Qi, and L. Bao, "Artificial-neural-network adaptive control of compound actuator," Journal of Beijing University of Aeronautics and Astronautics, vol. 33, no. 11, pp. 1295-1298, 2007.

[6] J. Zhou, C. J. Zhang, and C. Y. Wen, "Robust adaptive output control of uncertain nonlinear plants with unknown backlash nonlinearity," IEEE Transactions on Automatic Control, vol. 52, no. 3, pp. 503-509, 2007.

[7] R. Merzouki, J. A. Davila, L. Fridman, and J. C. Cadiou, "Backlash phenomenon observation and identification in electromechanical system," Control Engineering Practice, vol. 15, no. 4, pp. 447-457, 2007.

[8] G. Tao, X. Ma, and Y. Ling, "Optimal and nonlinear decoupling control of systems with sandwiched backlash," Automatica, vol. 37, no. 2, pp. 165-176, 2001.

[9] M. T. Mata-Jimenez, B. Brogliato, and A. Goswami, "On the control of mechanical systems with dynamic backlash," in Proceedings of the 36th Conference on Decision and Control, pp. 1990-1995, IEEE, Washington, DC, USA, 1997. 
[10] M. Nordin and P.-O. Gutman, "Controlling mechanical systems with backlash-a survey," Automatica, vol. 38 , no. 10, pp. 16331649, 2002.

[11] Z. Zuo, X. Li, and Z. Shi, "L 1 adaptive control of uncertain gear transmission servo systems with deadzone nonlinearity," ISA Transactions, vol. 58, pp. 67-75, 2015.

[12] S. Tarbouriech, I. Queinnec, and C. Prieur, "Stability analysis and stabilization of systems with input backlash," IEEE Transactions on Automatic Control, vol. 59, no. 2, pp. 488-494, 2014.

[13] P. Philipp, B. Thomas, B. Miroslav et al., "Hybrid approach to modeling, control and state estimation of mechanical systems with backlash," International Journal of Control, vol. 80, no. 11, pp. 1729-1740, 2007.

[14] Y.-J. Liu and S. Tong, "Adaptive fuzzy control for a class of nonlinear discrete-time systems with backlash," IEEE Transactions on Fuzzy Systems, vol. 22, no. 5, pp. 1359-1365, 2014.

[15] M. L. Corradini and G. Orlando, "Robust stabilization of nonlinear uncertain plants with backlash or dead zone in the actuator," IEEE Transactions on Control Systems Technology, vol. 10, no. 1, pp. 158-166, 2002.

[16] R. Dong and Y. Tan, "On-line identification algorithm and convergence analysis for sandwich systems with backlash," International Journal of Control, Automation and Systems, vol. 9, no. 3, pp. 588-594, 2011.

[17] Y. Tan and L. Deng, "Modeling the dynamic sandwich system with hysteresis using NARMAX model," Mathematics and Computers in Simulation, vol. 97, pp. 162-188, 2014.

[18] Z. G. Shi and Z. Y. Zuo, "Backstepping control for gear transmission servo systems with backlash nonlinearity," IEEE Transactions on Automation Science and Engineering, vol. 12, no. 2, pp. 752-757, 2015.

[19] R.-H. Du, Y.-F. Wu, W. Chen, and Q.-W. Chen, "Adaptive backstepping fuzzy control for servo systems with backlash," Control Theory \& Applications, vol. 30, no. 2, pp. 254-260, 2013.

[20] Z. Pengbing and S. Yaoyao, "Adaptive sliding mode control of the A-axis used for blisk manufacturing," Chinese Journal of Aeronautics, vol. 27, no. 3, pp. 708-715, 2014.

[21] V. Nekoukar and A. Erfanian, "Adaptive fuzzy terminal sliding mode control for a class of MIMO uncertain nonlinear systems," Fuzzy Sets and Systems, vol. 179, pp. 34-49, 2011.

[22] S. Yu, X. Yu, B. Shirinzadeh, and Z. Man, "Continuous finitetime control for robotic manipulators with terminal sliding mode," Automatica, vol. 41, no. 11, pp. 1957-1964, 2005. 


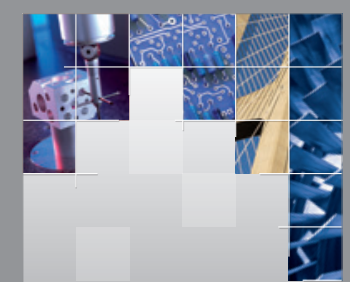

\section{Enfincering}
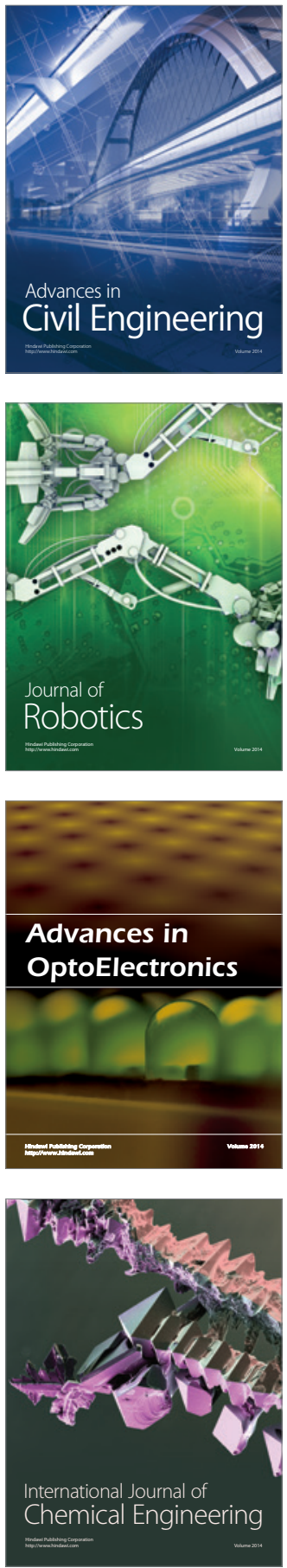

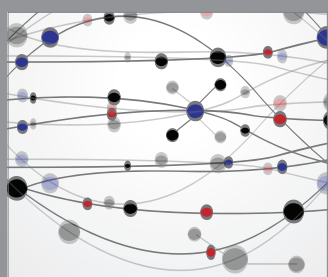

The Scientific World Journal

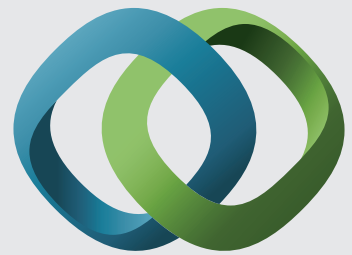

\section{Hindawi}

Submit your manuscripts at

https://www.hindawi.com
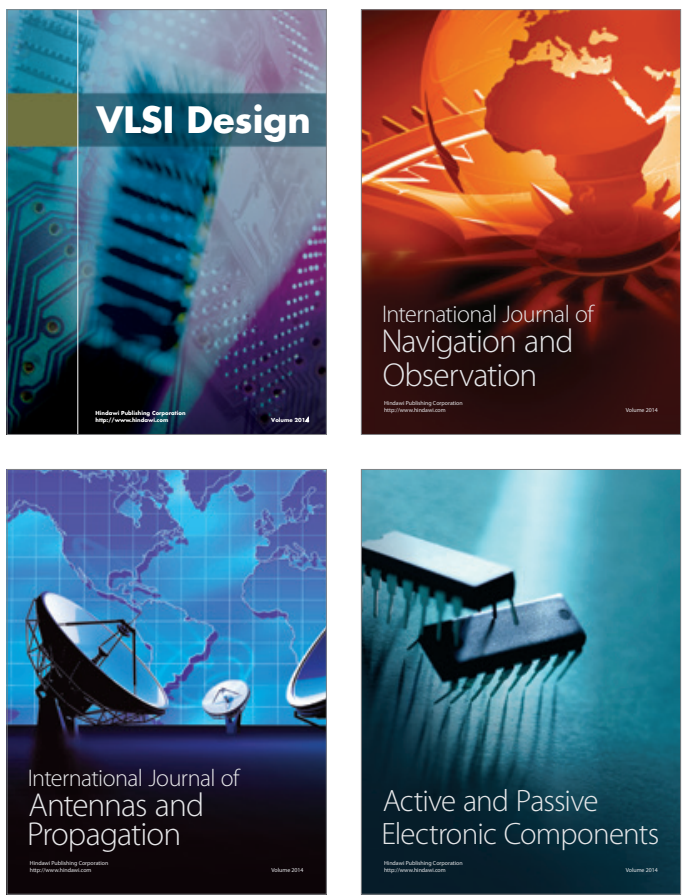
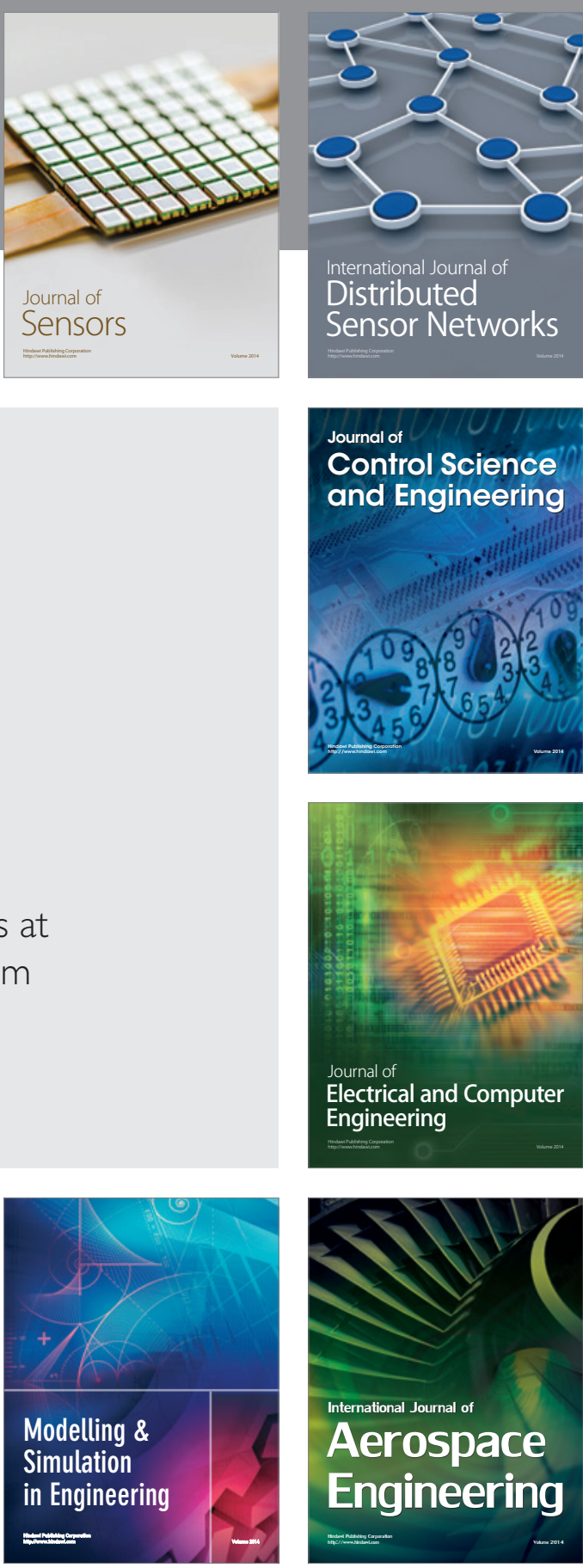

International Journal of

Distributed

Sensor Networks

$-$

Joumal of

Control Science

and Engineering
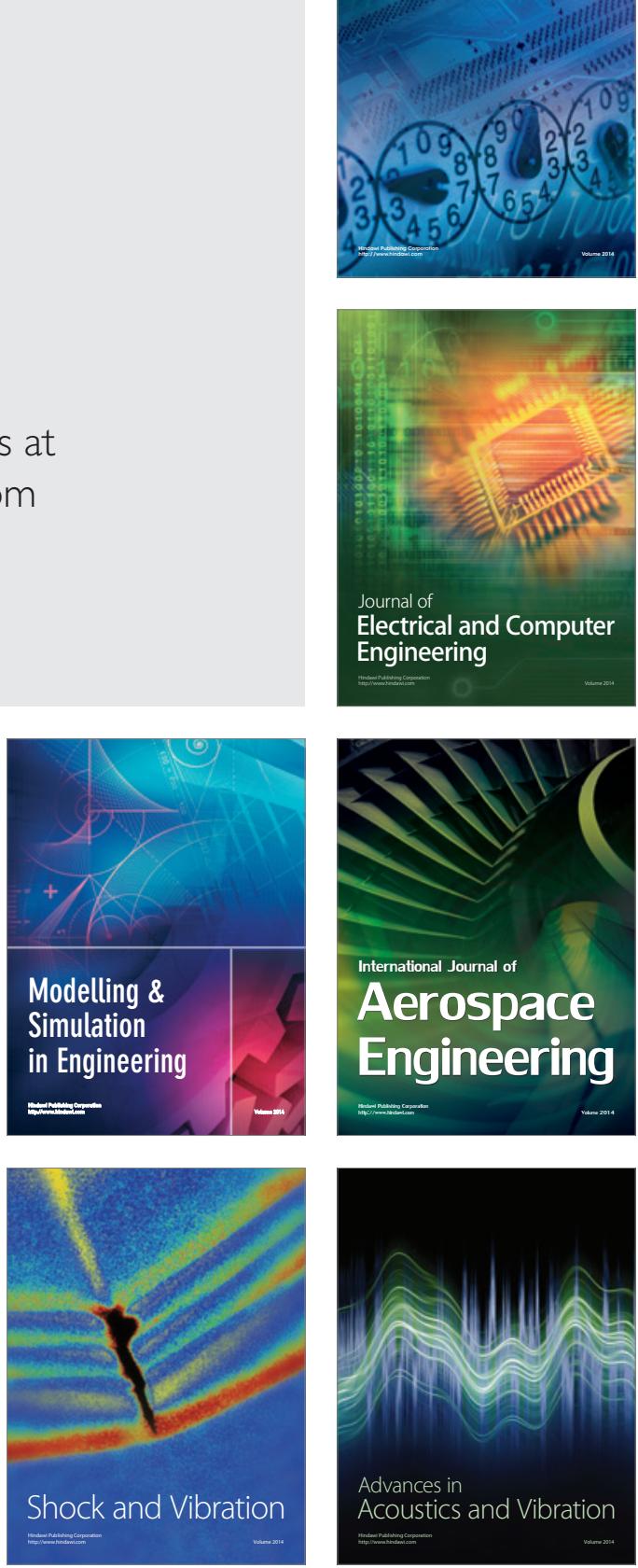